\title{
The utility of maternal mean platelet volume levels for early onset neonatal sepsis prediction of term infants
}

\author{
Yasemin Cekmez ${ }^{1}$, Merve Dizdar Güleçoğlu ${ }^{1}$, Cemile Özcan ${ }^{1}$, Leyla Karadeniz², Gürkan Kıran ${ }^{1}$ \\ ${ }^{1}$ Science Health University, Umraniye Medical and Research Hospital, Obstetrics Department, Turkey \\ ${ }^{2}$ Science Health University, Umraniye Medical and Research Hospital, Neonatology Department, Turkey
}

\begin{abstract}
Objectives: This study is aimed to evaluate the role of maternal mean platelet volume (MPV) levels for antenatal prediction marker of early onset neonatal sepsis in term infants born to mothers who have low infection risk.

Material and methods: A total of 62 pregnant women who gave birth in our hospital and whose neonates were admitted to a third level Neonatal Intensive Care Unit due to confirmed neonatal sepsis between January 2010 and May 2016 were selected as a study group. Within the same period, 68 women who gave birth to healthy neonates were enrolled as a control group. We compared maternal MPV values which were evaluated before delivery. The receiver operating characteristic (ROC) curves were drawn to evaluate the values maternal MPV in the diagnosis of neonatal sepsis.

Results: MPV levels were detected statistically higher in the study group than the control group ( $8.27 \pm 1.85$ vs. $8.98 \pm 1.16)$ $(p=0.001)$.

Conclusion: The maternal serum MPV level is a clinically useful, non-invasive and reliable marker in antenatal prediction of EOS.
\end{abstract}

Key words: early onset neonatal sepsis, mean platelet volum, pregnancy, infection

Ginekologia Polska 2017; 88, 6: 312-314

\section{INTRODUCTION}

Early onset neonatal sepsis (EOS) is a systemic inflammatory response syndrome occurring within 72 hours of birth [1]. Although lots of diagnostic measures and treatment approaches are available in developing countries, the incidence of EOS varies from 10 to 20/1000 live births and approximately $1 \%$ of infants die due to sepsis related causes [2].

Apnea, hypothermia, tachypnea, grunting, lethargy, and vomiting of an infant may put the clinician into doubt about EOS. These findings may often mixable with noninfective causes because they are not specific for EOS [3]. Verification of EOS is also important to avoid overtreatment of non-septic infants with antimicrobial agents which may cause to colonization with drug-resistant microorganisms and superinfection with other pathogens. At this point serum biomarkers have been proposed as being useful indicators for early identification of septic infants [4].

Many biomarkers have been reported to be valuable to predict the disease before clinical manifestations occuring in the literature [5-7]. But the common characteristics of these studies are either that they have examined the infants of high-risk mothers or have examined infants with suspicious clinical findings. In the recent literature there is no study evaluating maternal serum biomarkers to predict early neonatal sepsis in low risk mothers before the baby is born.

The objective of this study was to predict EOS in infants born to mothers who had low risk of infection. It is easy to predict the disease of infants born to mothers who had clinical findings of suspected or overt chorioamnionitis and any other signs of systemic infection, but neonatal sepsis may develop without any kind of maternal infection exist. Subclinical intrauterine infection has been implicated as a major etiological factor in the pathogenesis and subsequent morbidity associated with EOS developed due to preterm membrane rupture, but EOS can also be developed in infants born to mothers who had no risk factor of infection [8].

Due to the reason mentioned above, we aimed to evaluate the role of maternal mean platelet volume (MPV) levels 
for antenatal prediction marker of EOS in term infants born to mothers who have low infection risk.

\section{MATERIAL AND METHODS \\ Study population}

This retrospective observational study was performed in a tertiary referral hospital between January 2010 and May 2016 in İstanbul, Turkey. The institutional Ethics Committee approved the study protocol. Written informed consent was obtained from a parent or guardian of each infant. Women included in this study were divided into two groups as sepsis ( $n=62)$ and control $(n=68)$ according to whether their babies have taken EOS diagnosis. Babies suspected as EOS were admitted to our third level Neonatal Intensive Care Unit (NICU) and EOS were confirmed by the findings of blood and/or cerebrospinal fluid culture growing bacteria in NICU. The decision to obtain blood or cerebrospinal fluid for culture was at the discretion of the NICU clinicians.

Women with suspected or proven choriamnionitis clinical findings, any proven systemic infection and inflammation (either with higher WBC, CRP levels, fever or any other symptom of infection), preterm membrane ruptures, anemia, trombositopenia, platelet transfusion history, diabetes mellitus were excluded.

\section{Blood samples}

Five $\mathrm{ml}$ of venous blood was obtained from all participants at admission before any medication and IV fluid infusion performed. Only the blood samples taken no more than 5 hours prior to delivery were evaluated. MPV determinations were performed on the Coulter Counter model LH (Coulter Electronics, Hialeah, FL, USA). EOS diagnosis and follow-up of infants were done in our NICU unit by neaonatologist.

\section{Statistical analysis}

The statistical analyses were performed by using the Statistic Package for Social Sciences (ver. 12.0; SPSS Inc., Chicago, USA). Categorical variables were compared by Chi square test. Non-categorical variables were compared by Mann-Whitney test. The $p$ value $<0.05$ was accepted as statistically significant. ROC analysis was used to calculate a cut off value.

\section{RESULTS}

A total of 130 term singleton pregnant women were included in this study. The demographic characteristics were shown in Table 1. There was no difference between control and study groups in terms of mean maternal age ( $27 \pm 2$ vs. $28 \pm 1.1$ ), mean gestational weeks at admission $(39.26 \pm 1.15$ vs. $39.34 \pm 2.04)$ and mean birth weights ( $3450 \pm 290$ vs. $3380 \pm 310$ ). The groups were identical regarding the mode of delivery $(p=0.825)$.
Table 1. The demographic characteristics of groups

\begin{tabular}{|l|c|c|c|}
\hline & $\begin{array}{c}\text { Control } \\
(\mathbf{n = 6 8 )}\end{array}$ & $\begin{array}{c}\text { EOS } \\
(\mathbf{n = 6 2 )}\end{array}$ & $\mathbf{P}$ \\
\hline Age & $27 \pm 2$ & $28 \pm 1.1$ & 0.628 \\
\hline $\begin{array}{l}\text { Gravidity } \\
\text { Median (IQR) }\end{array}$ & $1(2)$ & $1(1)$ & 0.320 \\
\hline $\begin{array}{l}\text { Parity } \\
\text { Median (IQR) }\end{array}$ & $1(2)$ & $1(1)$ & 0.21 \\
\hline $\begin{array}{l}\text { Gestational weeks } \\
\text { Mean } \pm \text { SD }\end{array}$ & $39.26 \pm 1.15$ & $39.34 \pm 2.04$ & 0.788 \\
\hline $\begin{array}{l}\text { Delivery moden (\%) } \\
\text { vaginal } \\
\text { cesarean section }\end{array}$ & $\begin{array}{c}100(56.2 \%) \\
78(43.8 \%)\end{array}$ & $\begin{array}{l}27(57.4 \%) \\
20(42.6 \%)\end{array}$ & 0.876 \\
\hline
\end{tabular}

Comparison of the MPV and white blood cells (WBC) levels of the groups were reported in Figure1. There was no difference regarding WBC levels among groups ( $p=0.12$ ). MPV levels were detected statistically higher in study group than control group. $(8.27 \pm 1.85$ vs. $8.98 \pm 1.16 ; p=0.001)$ (Table 2).

None of the participants were thrombocytopenic but in comparison with the control group, platelet counts were lower in study group ( $264 \pm 25$ vs. $210 \pm 30 ; p=0.04$ ) (Table 2 ).

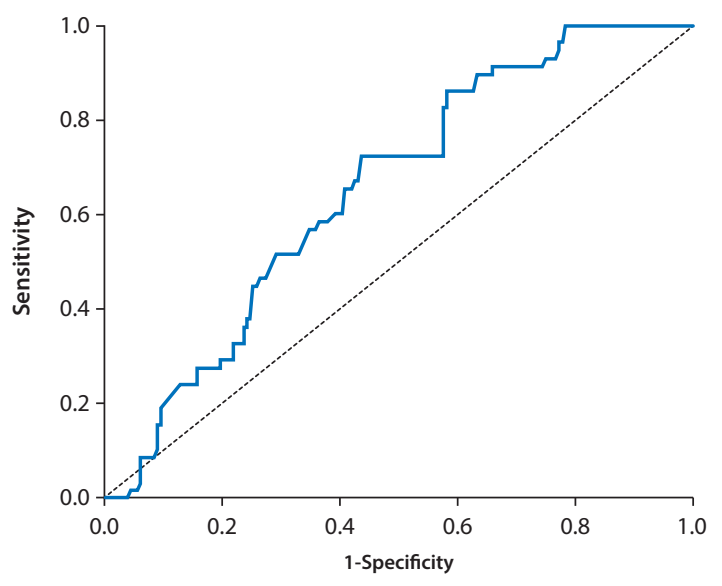

Figure 1. ROC diagram of MPV values

\begin{tabular}{|c|c|c|c|}
\hline & $\begin{array}{l}\text { Control } \\
(n=68)\end{array}$ & $\begin{array}{c}\text { EOS } \\
(n=62)\end{array}$ & $\mathbf{P}$ \\
\hline $\begin{array}{l}\text { WBC } \\
\text { Mean } \pm S D\end{array}$ & $27 \pm 2$ & $28 \pm 1.1$ & 0.628 \\
\hline $\begin{array}{l}\text { MPV } \\
\text { Mean } \pm \text { SD }\end{array}$ & $8.27 \pm 1.85$ & $8.98 \pm 1.16$ & 0.001 \\
\hline $\begin{array}{l}\text { Platelet count } \\
\text { Mean } \pm S D\end{array}$ & $264 \pm 25$ & $210 \pm 30$ & 0.04 \\
\hline
\end{tabular}


ROC analysis was used to calculate a cut off value but a significant cut off value was not found (AUC =0.65) (Fig. 1).

\section{DISCUSSION}

Early onset neonatal sepsis, mostly remarkable in the developing countries, is a major cause of morbidity and mortality of infants [1]. Early diagnosis and adequate theory of the infected neonates play a vital role in lowering such mortality and morbidity rates [4]. The best known risk factors for EOS are chorioamnionitis and maternal systemic infections. Preterm birth and preterm rupture of membranes are also other risk factors for chorioamnionitis and EOS [9].

Simple, rapid, non invasive, and safe intrauterine infection detection tests can be useful in prediction of neonatal infection of babies of mothers either with or without active labor. If maternal infections during pregnancy are diagnosed and treated early, the mortality and morbidity of neonates due to EOS can be decreased. In the literature, several diagnostic methods of peripartum intrauterine infection detection have been considered. These can be listed as amniotic fluid cultures, serum procalcitonin, C-reactive protein (CRP) interleukin (IL)-6, IL-8, IL-10, IL-18, tumor necrosis factor-alpha (TNF-alpha), interferon gamma levels etc. [10].

In all the studies we mentioned above, the authors aimed to diagnose the intrauterine infections of mothers who were in high risk for peripartum infections. The first difference of the present study was that we aimed to predict the EOS in women who were in low risk for peripartum infections. The second difference was that only term infants were included. Since the prematurity, preterm rupture of membranes and proven or suspected intrauterine infections are risk factors for EOS, we think that our strict inclusion and exclusion criterias are valuable in terms of the reliability of the prediction of EOS with maternal MPV levels. Due to the reasons mentioned above, even they have healthy babies, higher maternal MPV levels can be seen in those women excluded from the study.

In this study we selected the maternal MPV as a predictor of EOS asMPV is considered to be a marker of platelet production, consumption and severity of some diseases associated with bone marrow, hypoxia, perinatal inflammation and infections [11]. To our knowledge, the study is the first to report the high maternal MPV levels as a useful predictive marker of EOS diagnosis in term neonates born to mothers with low infection risk.

In concordance with our results there are studies reporting that high fetal MPV levels may predict the EOS in preterm infants $[12,13]$. These results are also valuable for early diagnosis of the disease of the neonates. The superiority of the present study is that it allows to predict the diagnosis of EOS before birth even in neonates born to women with low infection risk.

As a limitation of this study we did not analyze multi variation and demonstration of MPV as an independent risk factor for sepsis diagnosis. Another limitation was a small number of patients. Further analyses are needed to determine a cut-off value for MPV in such women with term singleton pregnancies.

In conclusion, analysis of maternal MPV is a simple laboratory investigation. In this study we found that mothers of term infants with EOS had higher maternal MPV levels before delivery. This might be associated with inflammatory and oxidative process.

\section{REFERENCES}

1. Gandhi S, Ranjan KP, Ranjan N, et al. Incidence of neonatal sepsis in tertiary care hospital: An overview. International Journal of Medical Science and Public Health. 2013; 2(3): 548, doi: 10.5455/ijmsph.2013.090320131.

2. Rasul $\mathrm{CH}$, Hassan MA, Habibullah M. Neonatal sepsis and use of antibiotic in a tertiary care hospital. Pakistan Journal of Medical Sciences. 2007; 23(1): 78-81.

3. Tripathi S, Malik GK. Neonatal Sepsis: past, present and future; a review article. Internet Journal of Medical Update - EJOURNAL. 2010; 5(2), doi: 10.4314/ijmu.v5i2.56163.

4. Lim WHo, Lien R, Huang YC, et al. Prevalence and pathogen distribution of neonatal sepsis among very-low-birth-weight infants. Pediatr Neonatol. 2012; 53(4): 228-234, doi: 10.1016/j.pedneo.2012.06.003, indexed in Pubmed: 22964280.

5. Fan Y, Yu JL. Umbilical blood biomarkers for predicting early-onset neonatal sepsis. World J Pediatr. 2012; 8(2): 101-108, doi: 10.1007/s12519012-0347-3, indexed in Pubmed: 22573419.

6. Saldir M, Tunc T, Cekmez F, et al. Endocan and Soluble Triggering Receptor Expressed on Myeloid Cells-1 as Novel Markers for Neonatal Sepsis. Pediatr Neonatol. 2015; 56(6): 415-421, doi: 10.1016/j.pedneo.2015.03.006, indexed in Pubmed: 26341458.

7. Cekmez F, Canpolat FE, Cetinkaya M, et al. Diagnostic value of resistin and visfatin, in comparison with C-reactive protein, procalcitonin and interleukin-6 in neonatal sepsis. Eur Cytokine Netw. 2011; 22(2): 113-117, doi: 10.1684/ecn.2011.0283, indexed in Pubmed: 21636351.

8. Çekmez Y, Çekmez F, Özkaya E, et al. Proadrenomedullin and serum amyloid $A$ as a predictor of subclinical chorioamnionitis in preterm premature rupture of membranes. J Interferon Cytokine Res. 2013; 33(11): 694-699, doi: 10.1089/jir.2012.0134, indexed in Pubmed: 24010826.

9. Oria de Rueda Salguero O, Beceiro Mosquera J, Barrionuevo Gonzalez M, et al. [Cord blood procalcitonin in the assessment of early-onset neonatal sepsis]. An Pediatr (Barc). 2016; 14, doi: 10.1016/j.anpedi.2016.09.003, indexed in Pubmed: 27751752.

10. Verstraete $\mathrm{EH}$, Blot $\mathrm{K}$, Mahieu $\mathrm{L}$, et al. Prediction models for neonatal health care-associated sepsis: a meta-analysis. Pediatrics. 2015; 135(4): e1002-e1014, doi: 10.1542/peds.2014-3226, indexed in Pubmed: 25755236

11. Cekmez F, Tanju IA, Canpolat FE, et al. Mean platelet volume in very preterm infants: a predictor of morbidities? Eur Rev Med Pharmacol Sci. 2013; 17(1): 134-137, indexed in Pubmed: 23329535.

12. Catal F, Tayman C, Tonbul A, et al. Mean platelet volume (MPV) may simply predict the severity of sepsis in preterm infants. Clin Lab. 2014; 60(7): 1193-1200, indexed in Pubmed: 25134389.

13. Ahmad MS, Waheed A. Platelet counts, MPV and PDW in culture proven and probable neonatal sepsis and association of platelet counts with mortality rate. J Coll Physicians Surg Pak. 2014; 24(5): 340-344, doi: 04.2014/JCPSP.340344, indexed in Pubmed: 24848393. 